\title{
Workshop de cocriação como ferramenta de design para inovação: Um estudo de caso sobre interfaces para carros autônomos
}

\author{
Co-creation workshop as a tool for innovative design: A case study on \\ interfaces for autonomous vehicles
}

\author{
QUARESMA, Manuela \\ LEUI | Laboratório de Ergodesign e Usabilidade de Interfaces, \\ Doutora em Design pela PUC-Rio \\ mquaresma@puc-rio.br \\ MOTTA, Isabela \\ LEUI | Laboratório de Ergodesign e Usabilidade de Interfaces, \\ Graduanda em Design pela PUC-Rio \\ isabela.canellas@gmail.com
}

\begin{abstract}
RESUMO
O rápido desenvolvimento de novas tecnologias está mudando a forma como usuários se relacionam com os produtos. Assim, é necessário que os designers repensem as interfaces de comunicação humanomáquina constantemente. A cocriação - processo de criação coletiva envolvendo usuários e desenvolvedores - é uma abordagem que oferece diversos benefícios para geração de propostas inovadoras. Este artigo tem como objetivo explorar a aplicabilidade de um workshop de cocriação na produção de propostas de interfaces para produtos derivados de sistemas de inteligência artificial, por meio de um estudo de caso sobre interfaces de carros autônomos. Para isso, foram realizadas duas sessões de um workshop de cocriação com alunos e professores de graduação e pós-graduação em Design, especialistas em design de interação e de informação, utilizando a técnica de brainstorming em grupo. Os resultados mostram que as interfaces propostas pelos designers foram diversificadas na forma de comunicação de informação, utilizando tecnologias existentes para apresentar mensagens de uma forma inovadora e adequada às necessidades dos usuários de carros autônomos.
\end{abstract}

Cocriação, carro autônomo, design de interface, brainstorming

\begin{abstract}
The increasingly growth in the development of new technology is changing the way uses Interact with products. Thus, it is necessary that designers rethink the human-machine interfaces for communicating information constantly. Co-creation - the process of joint creation of users and designers - is an approach to product and experience development that can substantially benefit the generation of innovative product proposals. The present paper aims to assess the feasibility of co-creation workshops for the development of proposals for interfaces of Artificial Intelligence derived products through a case study on interfaces for autonomous vehicles. For this purpose, two sessions of co-creation workshops using group brainstorming
\end{abstract}




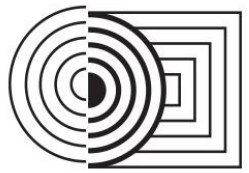

\section{$17^{\circ}$ ERGODESIGN \& USIHC 2019 \\ PUC-Rio, 11 a 13 de dezembro \\ Rio de Janeiro, RJ, Brasil}

$17^{\circ}$ Ergodesign - Congresso Internacional de Ergonomia e Usabilidade de Interfaces Humano Tecnológica: Produto, Informações Ambientes Construídos e Transporte

$17^{\circ}$ USIHC - Congresso Internacional de Ergonomia e Usabilidade de Interfaces Humano Computador

techniques were conducted with undergraduate and graduate Design students and professors, whom are specialists in interaction and information design. The results showed that the interfaces proposed by the designers were communicated information in a wide range of ways, making use of existing technologies to present messages in an innovative manner, meeting autonomous vehicles' users needs.

Co-creation, autonomous vehicle, interface design, brainstorming

\section{INTRODUÇÃO}

Sistemas de inteligência artificial vêm evoluindo e sendo capazes de realizar uma ampla gama de atividades. De acordo com David Coplin, CEO da Microsoft UK, "Essa tecnologia (Inteligência Artificial) irá mudar como nos relacionamos com a tecnologia" (INSIDER, 2016, tradução nossa). De fato, o avanço desses sistemas torna possível o desenvolvimento de produtos que podem revolucionar a maneira como os usuários realizam tarefas, como assistentes virtuais, robôs nas indústrias e carros autônomos, que já são uma realidade e cujo desenvolvimento evolui progressivamente.

Atualmente, assistentes de voz, como a Alexa, já contam com mais de 30 mil habilidades, permitindo que os usuários executem tarefas como fazer compras ou jogar jogos por meio da voz (STATISTA, 2018). Outro tipo de sistema dotado de inteligência artificial em expansão são os robôs, que devem revolucionar ainda mais o trabalho nas indústrias. Os CoBots - Robôs Colaborativos - já são capazes de reconhecer movimentos humanos e aprender novos comandos em segundos, sendo esperado que 150 mil unidades estejam em atividade até 2020 (TRADE MACHINES, 2019). Por fim, carros totalmente autônomos, que irão mudar a forma como motoristas se relacionam com automóveis, já estão sendo desenvolvidos e previstos para chegada ao mercado por volta de 2030 (FROST e SULLIVAN, 2018).

Os avanços na tecnologia de inteligência artificial estão modificando os produtos com os quais usuários realizam tarefas. Assim, esses avanços trazem também uma mudança na relação dos usuários com os produtos, alterando os papéis de usuários e sistemas na interação e as formas de comunicação de informações. Considerando isso, é fundamental que os designers de interação estejam atentos para essas mudanças, fazendo com que as interfaces dos produtos acompanhem as novas dinâmicas de interação humano-computador. Para realizar essa tarefa, os designers têm como ferramentas diversos métodos de criação. Este artigo busca apresentar os benefícios dos métodos de cocriação na geração de propostas de interfaces para produtos de inovação.

\section{A COCRIAÇÃO E O BRAINSTORMING NO PROCESSO DE INOVAÇÃO}

O termo cocriação pode ser amplamente entendido como o ato de criar coletivamente, ou seja, criatividade exercida por duas ou mais pessoas (SANDERS e STAPPERS, 2008). Atualmente, a palavra é comumente utilizada para descrever processos criativos nos quais profissionais responsáveis pelo desenvolvimento de produtos, interações, serviços, etc criam junto com os usuários e/ou outros atores da cadeira produtiva. Segundo Prahalad e Ramaswamy (2004), a cocriação se trata da criação de valor conjunta entre empresas e consumidores, da definição de problemas e da busca de soluções em colaboração, por meio do diálogo contínuo e em ambientes de experiência inovadora para novas experiências de cocriação.

Sanders e Stappers (2008) argumentam que, no processo de co-design, - entendido pelos autores como "a criatividade de designers e pessoas não treinadas em design trabalhando juntas no processo de desenvolvimento de design" (SANDERS e STAPPERS, 2008, p. 6, tradução 


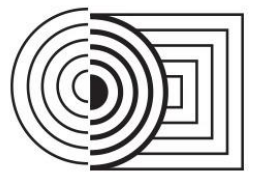

\section{$17^{\circ}$ ERGODESIGN \& USIHC 2019 \\ PUC-Rio, 11 a 13 de dezembro \\ Rio de Janeiro, RJ, Brasil}

$17^{\circ}$ Ergodesign - Congresso Internacional de Ergonomia e Usabilidade

de Interfaces Humano Tecnológica: Produto, Informações Ambientes

Construídos e Transporte

$17^{\circ}$ USIHC - Congresso Internacional de Ergonomia e Usabilidade

de Interfaces Humano Computador

nossa) - os atores do grupo têm novos papeis para desempenhar. O usuário final, que eventualmente vai se beneficiar do processo de design, é colocado na posição de expert de sua experiência. Já o pesquisador e o designer contribuem para o processo de geração de insights, dando suporte ao usuário com ferramentas para ideação e expressão, além de colaborar com suas habilidades de design no processo geração de ideias (SANDERS e STAPPERS, 2008).

Diversos exemplos de produtos e serviços ilustram o valor dos processos de cocriação para a inovação. Em 2008, a Starbucks desenvolveu o website MyStarbucksldea.com, uma plataforma na qual os consumidores da empresa poderiam compartilhar suas ideias e experiências com a Starbucks (RAMASWAMY, 2011). Todo mês, alguns funcionários revisavam e, se fossem adequadas, aplicavam algumas das ideias. De forma similar, a empresa francesa de telefonia, Orange, criou a plataforma IdClic, website onde os funcionários da empresa podiam compartilhar suas ideias (RAMASWAMY, 2011). No decorrer de cinco anos, foram geradas mais de 100 mil ideais, das quais 10 mil foram implementadas e geraram para a empresa mais de 900 milhões de euros em lucro e economias. Já a empresa de vidros e cerâmicas, Corning, além de unir seus engenheiros com usuários para desenvolver soluções para diferentes casos de uso, também firmou parcerias com diversas empresas, como a Sharp, a Volkswagen, e até com a Johns Hopkins University (TURIERA e CROS, 2013).

Existem diversas plataformas para exercer a cocriação, seja de forma virtual ou física. Em termos de plataformas de interação físicas, os workshops de design para cocriação podem trazer diversos benefícios. Os workshops de design são uma forma de design participativo que consolida os métodos de cocriação em sessões organizadas (MARTIN e HANINGTON, 2012). Esses workshops reúnem participantes - designers, usuários e outros atores da cadeia produtiva - para a realização de atividades criativas intensas, centradas na resolução de um problema, contribuindo para a ideação de soluções (MARTIN e HANINGTON, 2012). Segundo Martin e Hanington (2012), os workshops de design podem incluir ferramentas simples, permitindo que os participantes criem modelos ou desenhos para ilustrar soluções para um problema de design.

Em workshops de design, o Brainstorming em grupo é uma das técnicas de cocriação mais conhecidas e amplamente utilizadas. O Brainstorming é "um método individual ou em grupo para gerar ideais, aumentar a eficácia criativa ou achar soluções para problemas" (WILSON, 2013). Osborn (1962) afirma que o princípio do brainstorming consiste na suspensão do julgamento na geração de ideias: "(...) deve-se 'ligar' uma vez o espírito do julgamento e outra vez o de imaginação, em lugar de pensar simultaneamente, não só pelo aspecto crítico como imaginoso" (OSBORN, 1962, p. xxv). Segundo o autor, a geração de ideias livre de julgamento em um processo de brainstorming favorece a criatividade e o número de ideias geradas.

O procedimento básico do brainstorming em grupo consiste em quatro passos, segundo Wilson (2013, p. 14, tradução nossa):

1. Selecionar um grupo de três a dez participantes com diferentes backgrounds

2. Expor claramente um problema, questão ou tópico ao grupo

3. Pedir ao grupo para gerar soluções ou ideias sem crítica ou tentativas de limitar o tipo ou número de ideias

4. Discutir, criticar e, possivelmente, priorizar os resultados do brainstorming para etapas futuras no processo

O brainstorming em grupo apresenta diversos benefícios para a geração de ideias inovadoras, podendo beneficiar os novos produtos derivados de novas tecnologias. Em primeiro lugar, o brainstorming possibilita um grande número de ideias. Em processos criativos, a 


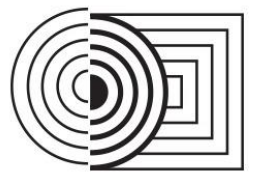

\section{$17^{\circ}$ ERGODESIGN \& USIHC 2019 \\ PUC-Rio, 11 a 13 de dezembro \\ Rio de Janeiro, RJ, Brasil}

$17^{\circ}$ Ergodesign - Congresso Internacional de Ergonomia e Usabilidade

de Interfaces Humano Tecnológica: Produto, Informações Ambientes

Construídos e Transporte

$17^{\circ}$ USIHC - Congresso Internacional de Ergonomia e Usabilidade

de Interfaces Humano Computador

quantidade de ideias geradas é fundamental porque as primeiras ideias geralmente não são as melhores (OSBORN, 1962). O autor ainda coloca que apenas $10 \%$ das ideias geradas em processos de ideação têm qualidade. Assim, o brainstorming traz vantagens para o processo criativo porque o acúmulo de ideias conduz à geração de mais ideias, levando, eventualmente, à uma solução (OSBORN, 1962).

Outro benefício do brainstorming em grupo é que a ideação coletiva tende a aumentar o poder associativo dos participantes (OSBORN, 1962). Ainda, segundo o autor, o trabalho em grupo dobra a quantidade de ideias geradas em relação a processos individuais. Além disso, estudos mostraram que a rivalidade existente na criação por grupos aumenta em cerca de $50 \%$ a eficácia do trabalho mental. Por fim, de acordo com Kelley e Littman (2005) apud Chammas (2018), o Brainstorming pode ser um ponto de partida para a inovação por ser divertido, liberar energia e ser capaz de gerar resultados com rapidez.

Considerando o exposto acima, é possível argumentar que o desenvolvimento de propostas para produtos da inovação, como sistemas de inteligência artificial, pode tirar proveito da criação colaborativa. Utilizando a abordagem da cocriação, o workshop de design como plataforma facilitadora de interação e o Brainstorming em grupo como técnica de criação colaborativa, este artigo apresenta um estudo de caso sobre a criação de interfaces para carros autônomos e como esses produtos podem se beneficiar dos métodos de cocriação.

\section{O CASO DOS CARROS AUTÔNOMOS}

O erro humano na condução de veículos é um grande problema para a segurança viária. No mundo, acidentes de carro matam cerca de 1,3 milhão de pessoas por ano (COSTLOW, 2018) sendo $90 \%$ desses acidentes causados por erro humano (GOLD et al., 2013). Considerando esse cenário, a implementação futura de carros autônomos, nos quais o sistema automatizado de direção é responsável por conduzir o veículo, é uma alternativa fundamental para a segurança de motoristas e pedestres e para prevenir prejuízos à sociedade.

Apesar dos grandes benefícios potenciais de carros autônomos, ainda existem algumas questões relacionadas a fatores humanos no uso dessas tecnologias. Isso acontece porque, com o nível de desenvolvimento atual, ainda não é possível retirar toda a responsabilidade do ser humano. Esse tipo de veículo, com automação condicional (ou nível 3), faz do motorista um usuário que deve estar receptivo para a necessidade de retomar o controle do veículo em determinadas situações (SAE, 2013). Dessa forma, os papéis na interação mudam, uma vez que o usuário não desempenha mais o papel de um motorista convencional, já que não realiza a tarefa de direção o tempo todo, mas também não é um passageiro, porque não está isento da responsabilidade de intervir.

O problema que surge a partir dessa nova dinâmica de interação é que, para realizar a tarefa de direção, o motorista deve estar no chamado loop de tomada de decisão (MERAT et al., 2018). Merat et al. (2018) propõe que, para estar no loop de tomada de decisão, o motorista precisa estar no controle físico do veículo (controlando volante, pedais etc) e monitorando as mudanças dinâmicas no ambiente de direção, incluindo as ações relacionadas ao sistema automatizado (MERAT et al., 2018). No entanto, o que surge é a tendência dos usuários a superestimarem as capacidades do sistema, confiando que o veículo irá lidar com todas as situações (GOLD et al, 2015). Essa confiança excessiva pode levar os usuários a ficarem complacentes, se distraindo da tarefa principal de direção e, até mesmo, realizando tarefas secundárias (BOROJENI et al., 2016). Essas ações podem levar o motorista a sair do loop de tomada de decisão, o que afeta a qualidade da sua retomada de controle (ZEEB et al., 2015; LOUW \& MERAT, 2017). 


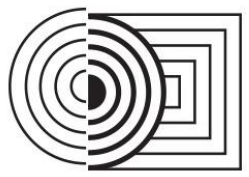

\section{$17^{\circ}$ ERGODESIGN \& USIHC 2019 \\ PUC-Rio, 11 a 13 de dezembro \\ Rio de Janeiro, RJ, Brasil}

$17^{\circ}$ Ergodesign - Congresso Internacional de Ergonomia e Usabilidade

de Interfaces Humano Tecnológica: Produto, Informações Ambientes

Construídos e Transporte

$17^{\circ}$ USIHC - Congresso Internacional de Ergonomia e Usabilidade

de Interfaces Humano Computador

Essa nova dinâmica entre motorista e veículo já se provou problemática. Em maio de 2016, ocorreu o primeiro acidente fatal envolvendo a condução de um carro autônomo, um Tesla Model S (BURNS e SHULGAN, 2018). O motorista estava utilizando a capacidade de "Autopilot" do Tesla Model S, lançada sete meses antes do acidente acontecer. O lançamento dessa funcionalidade gerou controvérsia porque o sistema não deixava claro que o motorista deveria continuar prestando atenção na via depois de ligar o sistema. Segundo investigações sobre o acidente, o sistema não foi capaz de reconhecer um caminhão que cruzava a pista. No entanto, foi observado que o caminhão poderia ter sido identificado pelo motorista cerca de 10 segundos antes do impacto, dando tempo a ele de retomar o controle. A falta de indícios de movimentos no volante ou frenagens apontou que o motorista não tomou nenhuma ação para desviar do caminhão, indicando que ele estava tão confiante no "Autopilot" que não estava prestando atenção à via (BURNS e SHULGAN, 2018).

As questões relacionadas aos carros autônomos estão vinculadas a diversos fatores, desde comportamentos humanos até tecnologia de hardware e software. No entanto, é evidente que a falha na apresentação de informações sobre o sistema automatizado pode ser fatal para usuários desses veículos. A apresentação adequada de informações para o motorista, contudo, pode não só amenizar os efeitos dos fatores humanos na direção autônoma, como, também, pode ajudar a melhorar o desempenho dos usuários na interação com esses sistemas. Para isso, a NHTSA, órgão regulador de trânsito dos EUA, recomenda que sejam apresentadas cinco informações básicas na interface humano-computador dos carros autônomos. Essas informações são sobre o status do sistema e o alerta sobre a necessidade de retomada de controle do sistema para o motorista (NHTSA, 2016).

Os carros autônomos com automação condicional são um exemplo de como os avanços da tecnologia mudam drasticamente uma tarefa realizada há décadas pelos seres humanos: dirigir. Como foi observado anteriormente, essa mudança exige que as interfaces projetadas para esses sistemas acompanhem a nova dinâmica de interação e sejam eficientes na apresentação de informação. Dessa forma, é necessário pensar em novas formas de apresentar informações sobre status de funcionamento dos sistemas automatizados de direção e alertas de retomada de controle para os motoristas por meio de uma interface. Considerando os benefícios da criação em grupo, este estudo de caso buscou gerar propostas de interfaces para carros autônomos em duas sessões de workshop para a cocriação com designers.

\section{METODOLOGIA}

Para este estudo de caso, foi conduzido um workshop de cocriação com professores e alunos de graduação e pós-graduação do Departamento de Artes e Design da PUC-Rio. O workshop teve como objetivo promover a criação de formas de apresentação de mensagens sobre status de funcionamento e de alertas de retomada de controle de sistemas de direção automatizados nível 3. Essa técnica foi escolhida porque permitiria a criação coletiva de soluções por especialistas em Design. A participação foi voluntária e não contou com nenhuma compensação financeira. O recrutamento foi feito a partir de um formulário que pediu informações básicas de contato e disponibilidade de horários, enviado por e-mail para os professores e por mídias sociais para os alunos.

Como se trata de um produto distante do mercado brasileiro, ainda não há "usuários" ou "clientes" reais para carros autônomos. No entanto, todos os participantes do workshop eram motoristas e/ou passageiros eventuais de automóveis, sendo considerados usuários em potencial desses veículos e capazes de se colocar na posição de usuários. Dessa forma, por um lado, as sessões 


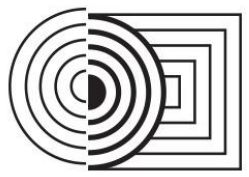

\section{$17^{\circ}$ ERGODESIGN \& USIHC 2019 \\ PUC-Rio, 11 a 13 de dezembro \\ Rio de Janeiro, RJ, Brasil}

$17^{\circ}$ Ergodesign - Congresso Internacional de Ergonomia e Usabilidade

de Interfaces Humano Tecnológica: Produto, Informações Ambientes Construídos e Transporte

$17^{\circ}$ USIHC - Congresso Internacional de Ergonomia e Usabilidade

de Interfaces Humano Computador

permitiram que os designers propusessem interfaces baseadas em seus conhecimentos de Design, e, ao mesmo tempo, pensassem nas suas necessidades como usuários de veículos em geral, trazendo suas experiências para as propostas. Por fim, a Universidade foi escolhida como ambiente para a realização das sessões de workshop porque é uma instituição que reúne, em um mesmo local, diversos especialistas em Design que lidam diariamente com projetos de inovação, de comunicação de informação e de pesquisa em Design.

\subsection{Procedimentos}

Foram realizadas duas sessões do workshop, nos dias 20/05/2019 e 22/05/2019, cada uma com duração de uma hora e meia. No total, compareceram oito participantes na primeira sessão (3 professores, 2 alunos de pós-graduação e 3 alunos de graduação) e nove na segunda sessão (6 professores, 1 aluno de pós-graduação e 2 alunos de graduação). O número de participantes está de acordo com o sugerido por Wilson (2013), que coloca que sessões de brainstorming em grupo devem ter de três a dez participantes. As duas sessões foram mediadas por duas pesquisadoras.

Os participantes chegaram ao local no horário marcado, assinaram o termo de consentimento livre e esclarecido e se acomodaram de forma confortável. Então, os participantes foram relembrados sobre o que era a pesquisa e qual era o objetivo do workshop. Uma breve apresentação foi feita para expor os conceitos de carro autônomo, os níveis de automação, o cenário de pesquisa e as questões acerca da apresentação de informação em carros autônomos. Foi, então, colocado o objetivo do workshop: "Como informar o motorista de um carro autônomo nível 3 sobre essas informações (recomendações da NHTSA)?". As duas sessões foram planejadas e executadas com estruturas diferentes entre si com o objetivo de obter uma maior variedade de resultados nas propostas geradas pelos participantes. Um resumo da estrutura dos dois dias de workshop pode ser encontrado no quadro 1.

$\mathrm{Na}$ primeira sessão, após a apresentação, foi realizada numa primeira etapa da sessão um brainstorming individual, na qual os participantes tiveram 15 minutos para elaborar, sozinhos, 0 máximo possível de propostas para a apresentação das mensagens aos motoristas. Após esse tempo, os participantes tiveram mais 20 minutos para gerar novas propostas em grupo. Foi incentivado que os participantes compartilhassem e discutissem sobre suas propostas elaboradas na primeira etapa para a elaboração de novas propostas. A etapa de refinamento e combinação de ideias foi inserida no workshop com base nas recomendações de Osborn (apud CHAMMAS, 2018). Ao fim do tempo de 20 minutos, a sessão de brainstorming chegou ao fim e os participantes tiveram 30 minutos para revisar as ideias propostas e discutir quais ideias, do ponto de vista do design de informação e da comunicação visual, seriam mais adequadas e porquê. Por fim, o workshop foi concluído agradecendo a participação de todos e as propostas de ideias foram recolhidas para análise futura. 


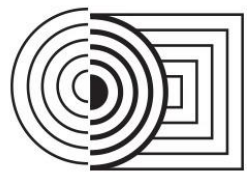

$17^{\circ}$ ERGODESIGN \& USIHC 2019

PUC-Rio, 11 a 13 de dezembro

Rio de Janeiro, RJ, Brasil $17^{\circ}$ Ergodesign - Congresso Internacional de Ergonomia e Usabilidade de Interfaces Humano Tecnológica: Produto, Informações Ambientes Construídos e Transporte

$17^{\circ}$ USIHC - Congresso Internacional de Ergonomia e Usabilidade de Interfaces Humano Computador

Quadro 1 - Estrutura das sessões do workshop

\section{Etapa}

Dia 1

$$
\text { Brainstorming individual }
$$

Brainstorming coletivo
3

1

2

\begin{tabular}{l|l}
1 & Brainstorming individual \\
2 & Brainstorming coletivo \\
3 & $\begin{array}{l}\text { Discussão das propostas e sua } \\
\text { adequação ao objetivo }\end{array}$
\end{tabular}

Dia 2

\section{Brainstorming em dupla}

Apresentação e discussão das ideias geradas na etapa 1

Tempo adicional para o detalhamento e representação das propostas (em dupla)

O segundo dia de workshop contou com uma estrutura diferente. O brainstorming inicial foi feito em duplas ao invés de individualmente, para incentivar a discussão na criação das ideias. Foi observado, no primeiro dia, que a segunda rodada de geração de ideias (coletiva) resultou apenas na exposição das soluções criadas e não na criação de novas ideias coletivamente. Por esse motivo, a segunda etapa de geração de ideias coletiva foi substituída pela exposição das ideias geradas em dupla e a etapa de discussão final foi substituída por um tempo adicional para os participantes refinarem e representarem visualmente as propostas. Essa última etapa foi acrescentada porque foi observado nos resultados da sessão 1 que as representações das soluções tinham menor nível de detalhe e especificação de funcionamento das interfaces. Por esse motivo, a primeira sessão teve maior quantidade de ideias, com menor grau de detalhamento, e a segunda sessão teve menor quantidade de ideias, mas com maior grau de detalhamento e representação visual.

Conforme as recomendações de Osborn (1962), nas duas sessões do workshop, os participantes foram incentivados a produzir uma grande quantidade de ideias em detrimento de ideias com um grau alto de qualidade. O grupo também foi encorajado a falar toda e qualquer ideia que viesse à mente, independente de sua plausibilidade. Ainda, os participantes foram instruídos a não criticar as propostas colocadas pelo grupo.

\subsection{Análise das propostas}

As propostas geradas no workshop foram analisadas em três etapas:

1) Primeiro, foi feita uma revisão inicial das propostas para identificar, em uma abordagem bottom-up, como as ideias poderiam ser divididas. A partir dessa revisão, as ideias foram categorizadas em: propostas que especificavam apenas Onde a informação deveria ser apresentada; propostas que especificavam apenas Como a informação deveria ser apresentada; propostas que especificavam Onde e Como as informações deveriam ser apresentas e; propostas de interface que, além de definir o local e a forma de apresentação das informações, também consideravam uma Sequência na apresentação das informações propostas pela NHTSA (2016).

2) Uma vez categorizadas, as propostas de interface também foram classificadas de acordo com: o Tipo de Mensagem apresentada (Status de funcionamento ou alerta de retomada de controle); A modalidade de apresentação da informação (visual, auditiva ou tátil); A forma de apresentação das mensagens, quando aplicável e; o Local de apresentação da informação, quando adequado. A classificação das propostas também utilizou uma abordagem bottom-up. 
3) Após essa etapa, para fins de melhor visualização, registro e organização dessas ideias, as propostas foram registradas digitalmente em um modelo criado para esta pesquisa. $O$ modelo (vide figuras na próxima seção) foi criado a partir do levantamento de todos os locais de apresentação de informações identificados na etapa 2.

\section{RESULTADOS}

Foram geradas 38 propostas de interface no primeiro dia de workshop e 17 no segundo dia, totalizando 55 ideias. Como mencionado anteriormente, a diferença no número de propostas geradas foi causada pela estrutura diferente nos dois dias de workshop. Apesar de um menor número de ideias geradas no segundo dia, as propostas desenvolvidas pelos participantes foram mais detalhadas e elaboradas, tendendo a ter a interação pensada para mais de um tipo ou forma de informação a ser apresentada. Assim, essas ideias, pensadas em sequência, foram desdobradas em mais de uma proposta de interface. Considerando o desdobramento das propostas, exemplificado pelas figuras 1 e 2, o número de propostas sobe para 100, sendo 60 do primeiro dia e 40 do segundo.

Considerando os desdobramentos das ideias, as propostas se dividiram nas categorias da seguinte forma:

Quadro 2 - Número de ideias por categoria

\begin{tabular}{c|cccc} 
Categorias & Onde & Como & Onde e como & Sequência \\
\hline $\begin{array}{c}\text { Número de } \\
\text { ideias }\end{array}$ & 6 & 11 & 32 & 51
\end{tabular}

Em relação ao tipo de mensagem, foi observado que 46 propostas trataram de alertas de retomada de controle, mostrando que o foco dos participantes foi em apresentar o alerta de retomada de controle de diversas maneiras. Em termos de modalidade de apresentação das mensagens, as propostas usavam as seguintes modalidades, ilustradas nas figuras 1, 2, 3 e 4:

Quadro 3 - Número de ideias por modalidade de apresentação da mensagem

\begin{tabular}{l|ccccccc} 
Modalidade & Visual & Auditiva & Tátil & $\begin{array}{c}\text { Auditiva } \\
\text { visual }\end{array}$ & Visual e tátil & $\begin{array}{c}\text { Visual, tátil e } \\
\text { auditivo }\end{array}$ \\
\hline $\begin{array}{l}\text { Número de } \\
\text { ideias }\end{array}$ & 62 & 6 & 11 & 14 & 2 & 5
\end{tabular}

De forma geral, a maioria das mensagens propostas foi elaborada para ser comunicada pelo canal visual. O uso de diferentes modalidades de apresentação também foi sugerido, principalmente, pelas propostas que apresentavam alertas de retomada de controle. Dentre as propostas de interface para alerta de retomada de controle, algumas ideias sugeriram o uso de alertas em mais de um momento, variando a urgência do alerta com a combinação de mais de uma modalidade para alertas mais urgentes. Algumas propostas de interface também sugeriram 


\section{$17^{\circ}$ ERGODESIGN \& USIHC 2019}

PUC-Rio, 11 a 13 de dezembro

Rio de Janeiro, RJ, Brasil $17^{\circ}$ Ergodesign - Congresso Internacional de Ergonomia e Usabilidade de Interfaces Humano Tecnológica: Produto, Informações Ambientes Construídos e Transporte

$17^{\circ}$ USIHC - Congresso Internacional de Ergonomia e Usabilidade de Interfaces Humano Computador

o uso de mais de um canal de apresentação de informação para mensagens sobre falha do sistema (figura 4). Para mensagens multimodais, a maioria das propostas misturou a visual com uma segunda modalidade, sendo auditiva ou tátil.

Figura 1. Representação da proposta 7. A proposta apresenta a informação de que o sistema está acionado por meio do display central e pela projeção no para-brisa. As informações são apresentadas visualmente e por avisos de voz.

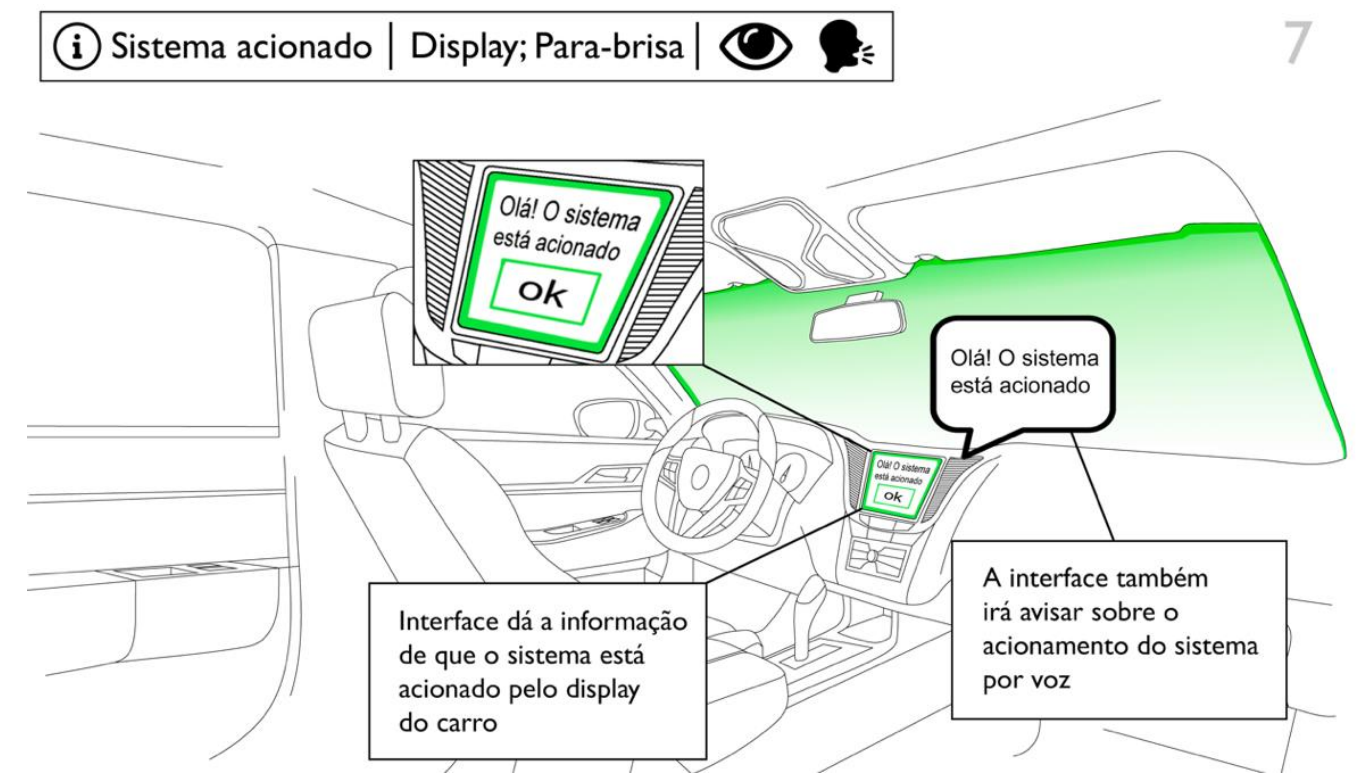

Figura 2. Desdobramento da proposta 7 (7.1). A proposta apresenta a informação de que o sistema está falhando por meio do display central e pela projeção de cor no para-brisa. As informações são apresentadas visualmente e por avisos de voz.

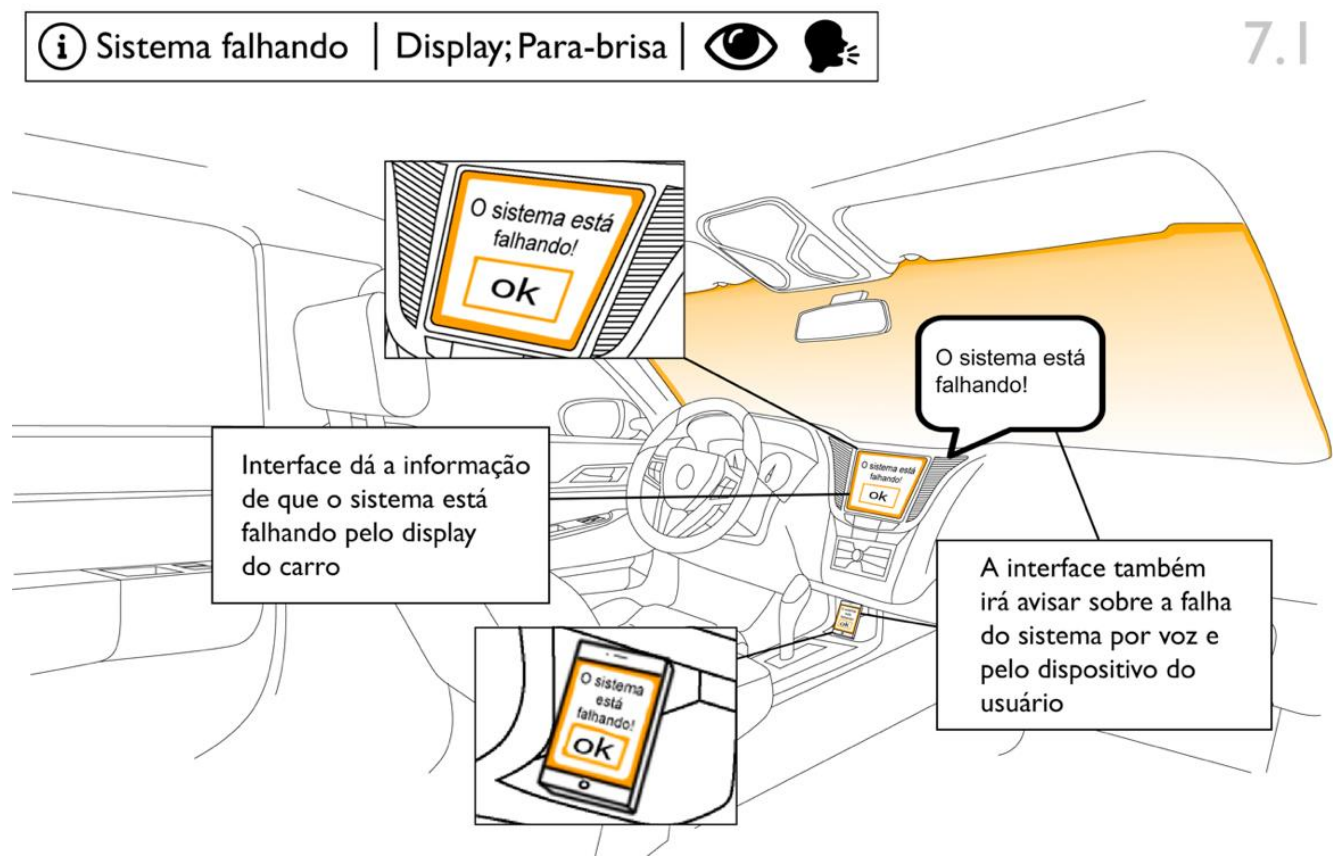


Figura 3. Proposta 2. A proposta apresenta um alerta de retomada de controle por meio de um ícone sonoro.

\section{(i) Retomada de controle $\mid$ (j))}

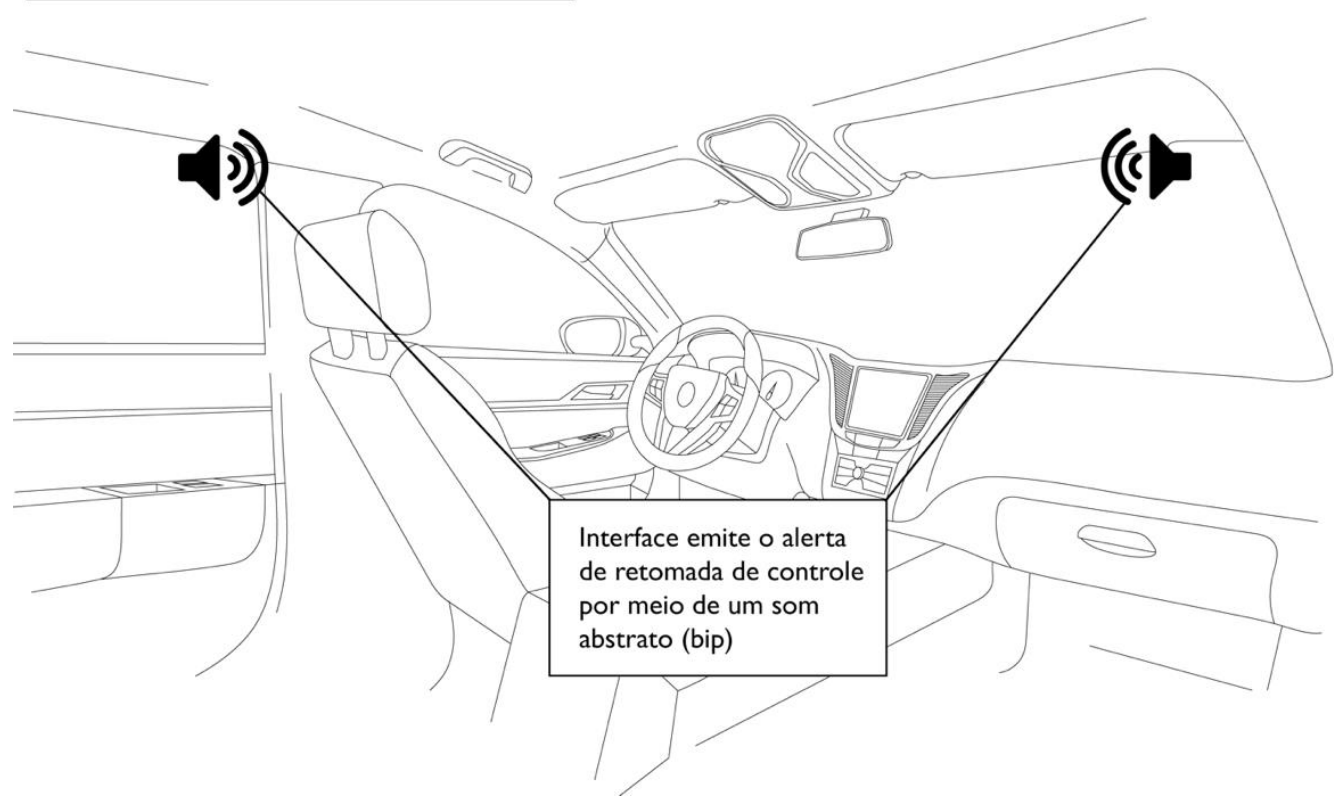

Figura 4. Proposta 8. A proposta apresenta um alerta de retomada de controle por meio de vibrações no banco do motorista e piscando as luzes internas do veículo.

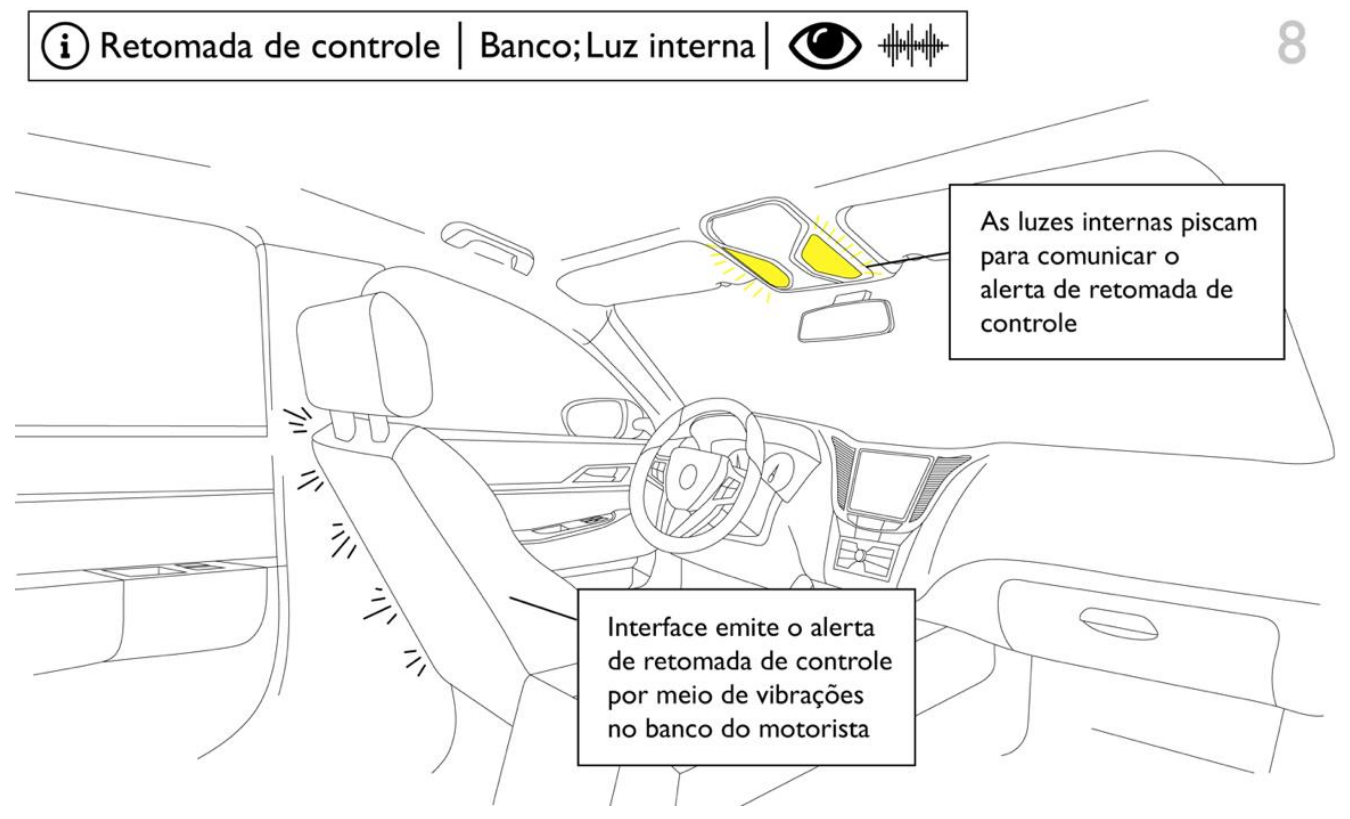

Os locais de apresentação de informação presentes nas propostas dos participantes do workshop foram: Banco do motorista; Volante; Painel; Display central; Para-brisa para projeção de HUD (head-up display); Espelho retrovisor; Luzes internas do veículo; Estrutura interna do 
$17^{\circ}$ ERGODESIGN

\& USIHC 2019

PUC-Rio, 11 a 13 de dezembro

Rio de Janeiro, RJ, Brasil $17^{\circ}$ Ergodesign - Congresso Internacional de Ergonomia e Usabilidade de Interfaces Humano Tecnológica: Produto, Informações Ambientes Construídos e Transporte

$17^{\circ}$ USIHC - Congresso Internacional de Ergonomia e Usabilidade

de Interfaces Humano Computador

carro; Óculos de Realidade Virtual para o motorista; Smartphone do motorista e; Câmbio. A figura 5 mostra o número de ideias propostas em cada um desses locais:

Figura 5. Número de vezes que os locais de apresentação de informação foram proposto nas ideias dos participantes

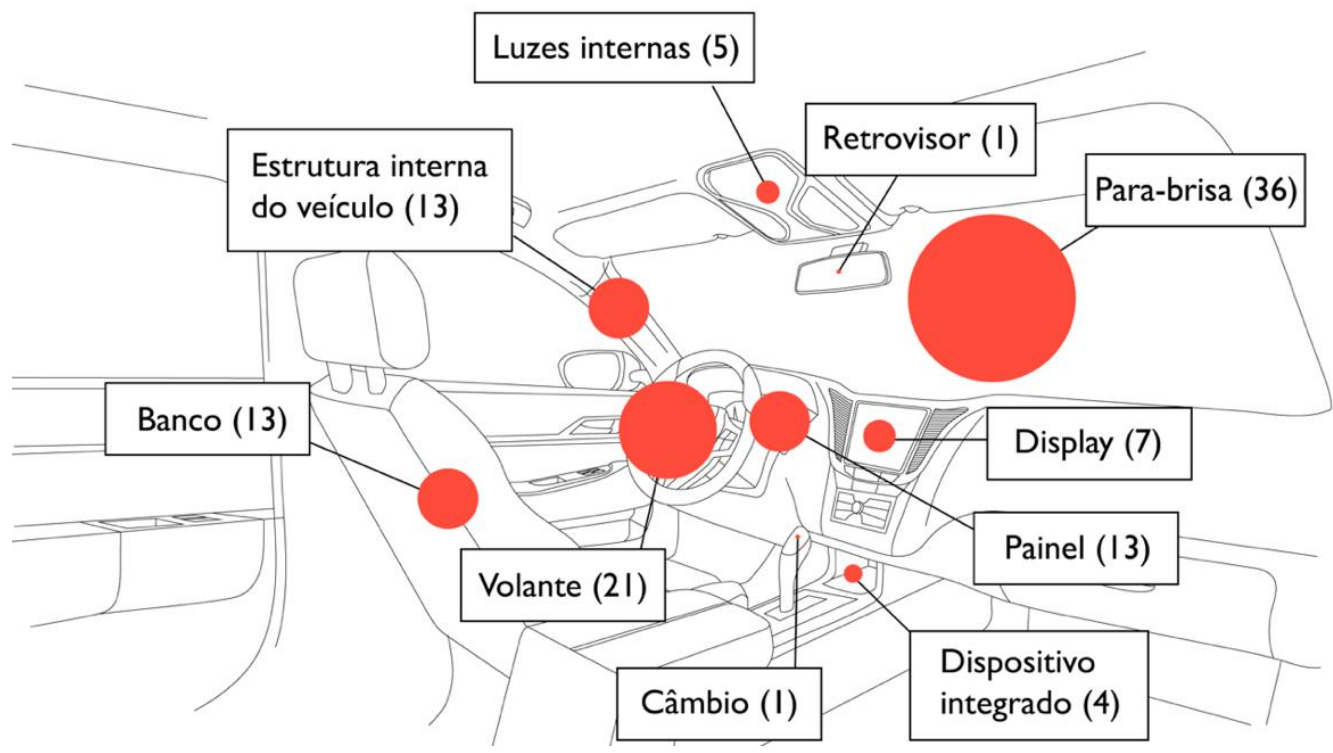

Além das classificações por tipo, modalidade e local de apresentação da mensagem, as propostas também foram classificadas em relação a forma como as informações foram apresentadas. Foi possível identificar a formatação de mensagens visuais por meio de: ícones; código de cores (exemplos na figura 6); texto e números; iluminação periférica e; frequência (elementos que piscam, exemplos nas figuras 7 e 8). Para as mensagens auditivas, as formas de apresentação foram: por voz; por meio de um ícone sonoro (som abstrato) (BLATTNER et al., 1989) e; por meio de ícones auditivos, sons que imitam os sons produzidos por elementos reais para representar uma interação (ex: buzina para alerta de retomada de controle) (ROGINSKA, 2013). Por fim, a apresentação de mensagens no canal tátil se limitou ao uso de vibrações. 
Figura 6. Proposta 33. Proposta de interface que comunica a probabilidade de um alerta de retomada de controle. A interface comunica essa informação por meio de ícones no para-brisa que representam o cenário da via. A interface usa código de cor para comunicar diferentes probabilidades de alertas.

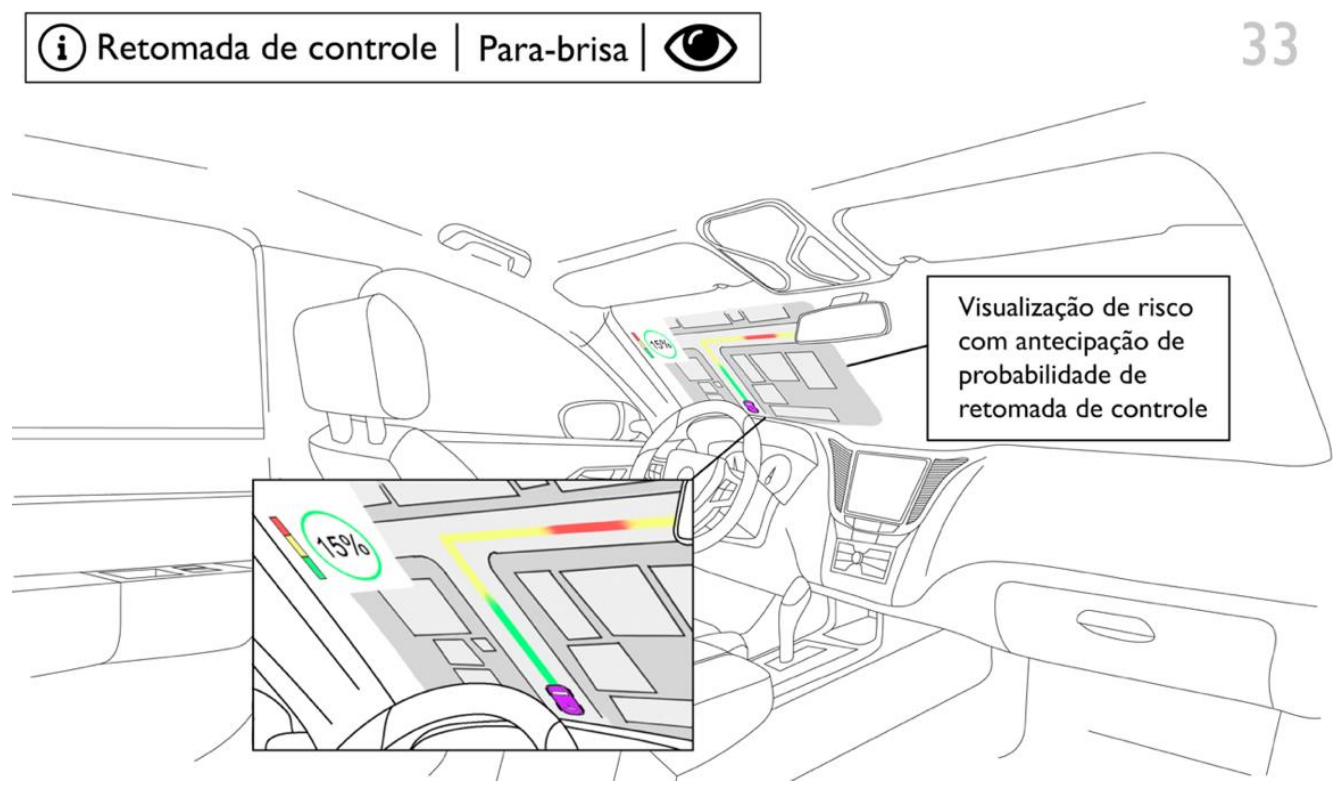

Figuras 7 e 8. Propostas 15.3 e 15.4. Variação de urgência da mensagem por meio de diferentes níveis de preenchimento com cor. Na figura da esquerda, a cor vermelha é utilizada apenas em algumas linhas do volante para comunicar uma falha no sistema. Já na figura da direita, o volante fica todo vermelho para comunicar um alerta de retomada de controle.

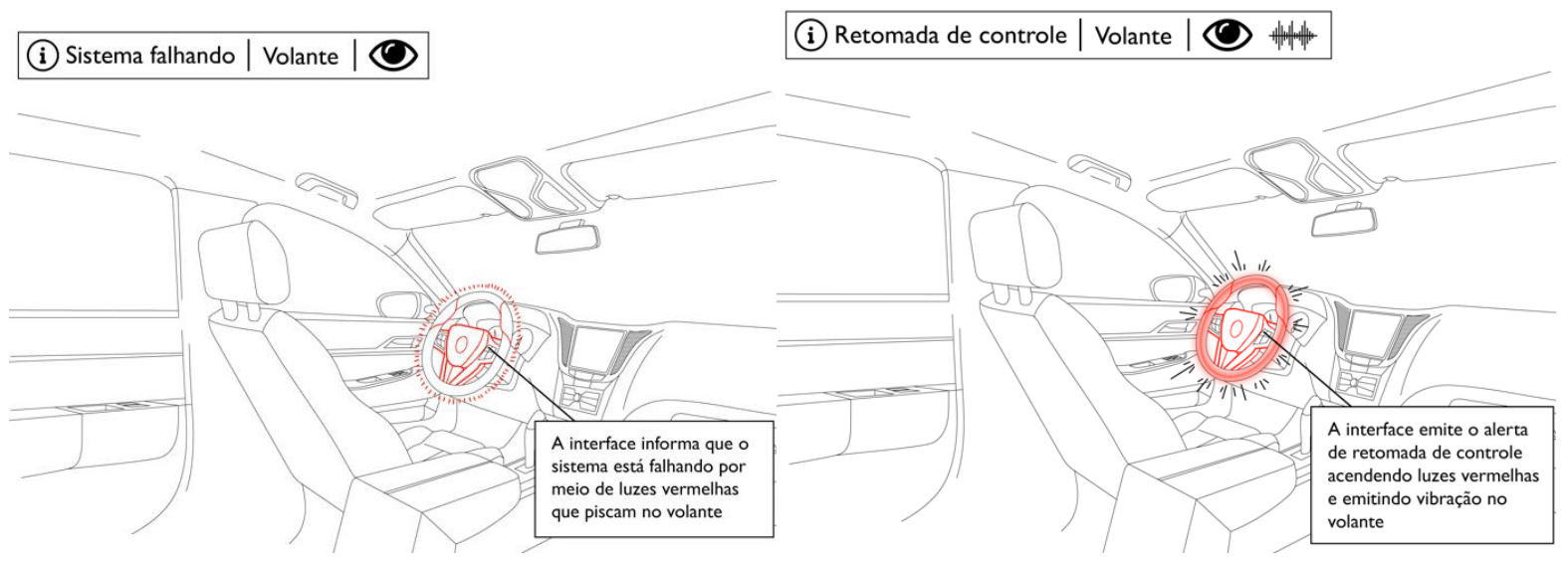

\section{CONCLUSÃO}

Devido ao avanço progressivo das novas tecnologias, como sistemas de inteligência artificial, as dinâmicas de interação entre usuários e produtos está mudando. Considerando essa tendência, os designers devem repensar as formas como projetam interfaces para os novos produtos, se adequando às necessidades dos usuários e às demandas de comunicação de informação dos produtos. Para projetar para as novas tecnologias, a cocriação é uma abordagem que traz diversas vantagens para o trabalho dos designers. Este artigo buscou avaliar os benefícios da cocriação por meio de um estudo de caso, que reuniu alunos e professores de graduação e pós- 


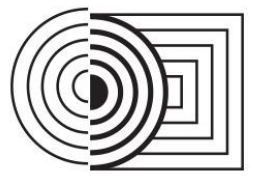

\section{$17^{\circ}$ ERGODESIGN \& USIHC 2019 \\ PUC-Rio, 11 a 13 de dezembro \\ Rio de Janeiro, RJ, Brasil}

$17^{\circ}$ Ergodesign - Congresso Internacional de Ergonomia e Usabilidade

de Interfaces Humano Tecnológica: Produto, Informações Ambientes Construídos e Transporte

$17^{\circ}$ USIHC - Congresso Internacional de Ergonomia e Usabilidade

de Interfaces Humano Computador

graduação de Design em duas sessões de um workshop de cocriação para a criação de propostas de interfaces para carros autônomos.

Considerando que os workshops contaram com estruturas diferentes, os resultados em termos de propostas também variaram. No primeiro dia, no qual os participantes tiveram duas rodadas de brainstorming, foi observado que o número de ideias geradas foi maior. No entanto, a representação gráfica dessas ideias foi menos detalhada e as interfaces propostas tiveram a tendência de serem pensadas para apresentar apenas uma informação (focando, principalmente, no alerta de retomada de controle). Dentre essas propostas, as formas de apresentação foram variadas, mas a qualidade das interfaces propostas foi inferior. Já no segundo dia da dinâmica, em que os participantes tiveram apenas uma rodada de brainstorming, foi observado uma queda no número de propostas. Porém, as ideias de interface geradas nesse dia tenderam a ser mais detalhadas em termos de representação e a serem pensadas para apresentar diversas informações, resultando em interfaces mais integradas na apresentação de informações.

Essa diferença na qualidade e quantidade das propostas entre os dois dias se deu pela variação de estrutura escolhida para os dois dias de sessão. Tais resultados mostram que é possível aplicar diferentes abordagens para obter resultados variados ao aplicar dinâmicas de cocriação. Apesar das diferenças nas ideias geradas, no entanto, um fato que foi constante nos dois dias de workshop foi a riqueza da produção em grupo. Nos dois dias, as discussões e a ideação em grupo contribuiu de forma significativa para o desenvolvimento das propostas, permitindo que os participantes trocassem informações sobre suas experiências como motoristas e passageiros e que gerassem ideias em conjunto. Além disso, a multiplicidade de pontos de vista e de conhecimentos na área de Design trouxe benefícios para o processo.

Ainda, apesar de os locais de apresentação dessas mensagens e a tecnologia utilizada para isso não serem novas, a forma como as propostas apresentaram as informações foi inovadora. Como ilustrado na figura 6, algumas das propostas dos participantes misturaram elementos já existentes, como rotas de um GPS e projeção no para-brisas, para apresentar uma informação nova (probabilidade de alerta de retomada de controle), de um jeito totalmente novo: prévisualização de risco sobreposto à rota do veículo. Além disso, também foi observado que as propostas buscaram respeitar os princípios do design, utilizando linguagens como ícones, códigos de cor e até outras modalidades de interação, além da visual.

Esses resultados mostram os benefícios de se reunir variados níveis de proficiência e habilitação em Design para desenvolver propostas de interface em grupo. Por serem detentores de conhecimento diversas áreas do Design, as propostas geradas, naturalmente, já consideravam os princípios de Design necessários para a apresentação de mensagens em uma interface. Ao mesmo tempo, por serem usuários em potencial desses carros autônomos, seja como passageiros ou motoristas, os participantes foram capazes de trazer suas experiências como usuários para as propostas. Dessa forma, as propostas geradas na dinâmica foram resultado de um processo criativo que combinou a experiência dos participantes com carros e seus conhecimentos acadêmicos e profissionais sobre o Design.

Por fim, é necessário ressaltar que o local escolhido para a realização dos workshops, a Universidade, trouxe grandes privilégios para o processo. Por ser uma instituição que trata com pesquisa e desenvolvimento de inovação e tecnologia de ponta de forma diária, os professores e alunos tiveram conhecimento sobre o que é possível e viável de ser feito. Ao mesmo tempo, por estarem acostumados com técnicas de criação e cocriação, como workshops de Design e brainstorming, os participantes foram capazes de gerar ideias sem críticas e julgamentos derivados de seus conhecimentos sobre aplicabilidade. Além disso, a Universidade é uma 


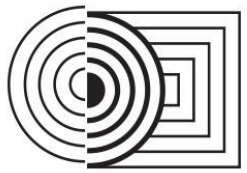

instituição que está livre de obrigações de mercado como prazos para o desenvolvimento de produtos, metas de vendas ou orçamentos. Utilizando métodos de produção alternativos e simulações, o desenvolvimento de pesquisa de inovação nas Universidades tem grande valor para o avanço do mercado.

\section{REFERÊNCIAS BIBLIOGRÁFICAS}

BLATTNER, M. M.; SUMIKAWA, D. A.; GREENBERG, R. M. Earcons and icons : their structure and common design principles. HUMAN-COMPUTER INTERACTION, V. 4, P. 11-44, 1989.

BOROJENI, S. S., e colab. Assisting Drivers with Ambient Take-Over Requests in Highly Automated Driving. In AutomotiveUI 2016 - 8th International Conference on Automotive User Interfaces and Interactive Vehicular Applications (pp. 237-244), 2016. p. 237-244.

BURNS, L. D., SHULGAN, C., Autonomy: The quest to build the driverless car - and how it will reshape our world. New York, New York, USA: HarperCollins, 2018.

CHAMMAS, A. S., QUARESMA, M., MONT'ALVÃO, C., A produção criativa de crianças no brainstorming de desenvolvimento de inovações em design de produtos digitais. Rio de Janeiro, 2018. 242p. Tese de Doutorado - Departamento de Artes \& Design, Pontifícia Universidade Católica do Rio de Janeiro.

COSTLOW, T., Detecting Pedestrians. Autonomous Vehicle Engineering, n. May, p. 68, 2018.

FROST \& SULLIVAN, GLOBAL AUTONOMOUS DRIVING MARKET OUTLOOK, 2018, 2018. Disponível em: https://info.microsoft.com/rs/157-gqe-382/images/k24a2018\%20frost\%20\%26\%20sullivan\%20-\%20global\%20autonomous\%20driving\%20outlook.pdf

Acesso em 12 de setembro de 2019.

GOLD, C., DAMBOCK, D., LORENZ, L., BENGLER, K., "Take over!” How long does it take to get the driver back into the loop?, In: Proceedings of the Human Factors and Ergonomics Society $57^{\text {th }}$ Annual Meeting, San Diego, USA, 2013.

GOLD, C., e colab. Trust in Automation - Before and After the Experience of Take-over Scenarios in a Highly Automated Vehicle. In Procedia Manufacturing (Vol. 3, pp. 3025-3032). 2015. p. 3025-3032.

INSIDER, MICROSOFT EXEC: 'AI IS THE MOST IMPORTANT TECHNOLOGY THAT ANYBODY ON THE PLANET IS WORKING ON TODAY', 2016. disponível em: https://www.insider.com/microsoft-exec-ai-is-the-most-important-technology-that-anybody-onthe-planet-is-working-on-today-2016-5 acesso em 12 de setembro de 2019.

LOUW, T., MERAT, N., Are you in the loop? Using gaze dispersion to understand driver visual attention during vehicle automation. Transportation Research Part C: Emerging Technologies, v. 76, p. 35-50, 2017.

MARTIN, B., HANINGTON, B., Universal Methods of Design: 100 ways to research complex problems. Beverly, MA: Rockport, 2012.

MERAT, N., et al., The "Out-of-the-Loop" concept in automated driving: proposed definition, measures and implications, In: Cognition, Technology \& Work, Springer, 2018.

NHTSA. Federal Automated Vehicles Policy: Accelerating the Next Revolution In Roadway Safety. U.S. Department of Transportation. [S.I: s.n.], 2016. 
OSBORN, A. F., O poder criador da mente: Princípios e processos do PENSAMENTO CRIADOR e do "BRAINSTORMING", São Paulo: IBRASA, 1962.

PRAHALAD, C. K. e RAMASWAMY, Venkat. Co-creation experiences: The next practice in value creation. Journal of Interactive Marketing, v. 18, n. 3, p. 5-14, 2004. Disponível em: $<\mathrm{http}: / /$ dx.doi.org/10.1002/dir.20015>.

RAMASWAMY, Venkat. Innovation through Co-creation: Engaging Customers and Other Stakeholders. 2011, Philadelphia, PA: William \& Philly's Mack Center for techological innovation, 2011. p. 5-7.

SANDERS, E., STAPPERS, P., Co-creation and the new landscapes of design. CoDesign, v. 4, n. 1, p. 5-18, 2008.

SOCIETY OF AUTOMOTIVE ENGINEERS INTERNATIONAL. Taxonomy and Definitions for Terms Related to Driving Automation Systems for On-Road Motor Vehicles. SAE International. Warrendale: [s.n.], 2018.

STATISTA, DIGITAL ECONOMY COMPASS 2018. 2018, Disponível em: https://static2.statista.com/download/pdf/digital economy compass 2018.pdf Acesso em 29 de abril de 2019.

TRADE MACHINES, GET READY FOR ROBOLUTION!, 2019, Disponível em: http://trademachines.com/info/robolution/ Acesso em 12 de setembro de 2019.

TURIERA, T., CROS, S., CO business: 50 examples of business collaboration. [S.I.]: Infonomia, 2013. Disponível em: <http://www.infonomia.com>.

WILSON, C., Brainstorming and Beyond. [S.I.]: Elsevier, 2013. Disponível em: $<$ https://linkinghub.elsevier.com/retrieve/pii/C20120035338>.

ZEEB, K., BUCHNER, A., SCHRAUF, M., What determines the take-over time? An integrated model approach of driver take-over after automated driving. Accident Analysis and Prevention, v. 78, p. 212-221, 2015.

\section{AGRADECIMENTOS}

Ao CNPq pelo financiamento da bolsa PIBITI. 